\title{
IUFOST2006/1361 Organisation of Plant Cell Wall by Imaging Techniques
}

\author{
F. Guillon ${ }^{\mathrm{a}}$, C. Barron ${ }^{\mathrm{b}}$, B. Bouchet ${ }^{\mathrm{a}}$, M.-F. Devaux ${ }^{\mathrm{a}}$, F. Jamme ${ }^{\mathrm{c}}$, S. Philppe ${ }^{\mathrm{d}}$, P. Robert ${ }^{\mathrm{e}}$, L. Saulnier ${ }^{\mathrm{a}}$ and \\ O. Tranquet ${ }^{\mathrm{a}}$ \\ ${ }^{a}$ INRA - Research Unit : Biopolymers, Interactions, Assemblies, Rue de la Géraudière BP-71627, F-44316 \\ Nantes, France

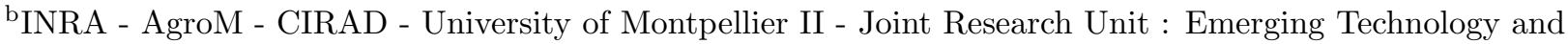 \\ Polymer Engineering, 2 Place Pierre Viala, 34060 Montpellier cedex 1, France \\ 'Synchrotron SOLEIL, L'Orme des Merisiers, BP 48, Saint Aubin, 91192 Gif sur Yvette, France \\ dInstitut National de la Recherche Agronomique, Rue de la Géraudière BP-71627, F-44316 Nantes, France \\ 'INRA - Research Unit : Biopolymers, Interactions, Assemblies, Rue de la Géraudière - BP 71627,44316 \\ Nantes cedex 3, France \\ guillon@nantes.inra.fr
}

Plant cell walls play a major role in plant texture of fruit and vegetable. In cereal, plant cell walls strongly influence cereal grain processing (milling, baking, malting ...) and quality of the end-product (for example texture and shelf-life of bread). In addition, cell walls are the major source of our dietary fibre. In planta, cell walls fulfil different functions: provision of strength and shape to cell, rigidity to the whole of plant. They are involved in the control of cell growth and protection from attack by pathogen and predators. They participates in cell to cell signalling. As a consequence, cell walls are not uniform. The type, size and shape of cell walls vary in close relation with the function of cell within a tissue. Cell walls are multi-component composites involving polysaccharides, structural proteins and in some cases, lignin. These biopolymers are held together by various intermolecular bonds. The nature of the biopolymers and their relative proportions change according to plant tissues and plant taxon. In addition, cell walls are remodelled in relation with cell development in several plant tissues. Complete understanding of the structure of plant cell walls has still to be achieved. Although the chemical structure of individual components of cell walls have been delineated, the way they interact and assemble to confer cell walls their original physical and physico-chemical properties are less well known. For many year, traditional methods of chemical analysis have yield information on bulk cell composition. However, such methods do not provide information of individual cells of plant tissues. This can achieved using microspectrocopic techniques and microscopic techniques. Both FT-IR and Raman microspectroscopy have been shown to be powerful to investigate in situ cell wall composition. Raman and FT-IR spectroscopy provide complementary information on molecular vibrations. While FT-IR spectroscopy mainly works with asymetrical vibrations of covalent bonds, Raman spectroscopy is more sensitive to the symetrical vibrations. However, conventional equipment, especially true for FT-IR microspectrometry due to the limited spatial resolution and spectral quality could not reveal chemical features within cellular dimensions. The use of synchrotron source for FT-IR microscopy, thanks to its brightness can overcome these limitations. Besides, the distribution of cell wall polysaccharides at the cell wall scale can be investigated using affinity probes, enzyme and derived carbohydrate binding modules and antibodies The presentation will demonstrate through the example of cereal grain how these different techniques can be applied to address the diversity in structural and chemical composition of cell walls in plant tissues and food systems. 